This item was submitted to Loughborough's Research Repository by the author.

Items in Figshare are protected by copyright, with all rights reserved, unless otherwise indicated.

\title{
Spectrum and energy efficiency in dynamic UAV-powered millimeter wave networks
}

PLEASE CITE THE PUBLISHED VERSION

https://doi.org/10.1109/LCOMM.2020.3001357

PUBLISHER

IEEE

VERSION

AM (Accepted Manuscript)

\section{PUBLISHER STATEMENT}

(c) 2020 IEEE. Personal use of this material is permitted. Permission from IEEE must be obtained for all other uses, in any current or future media, including reprinting/republishing this material for advertising or promotional purposes, creating new collective works, for resale or redistribution to servers or lists, or reuse of any copyrighted component of this work in other works.

\section{LICENCE}

\section{All Rights Reserved}

\section{REPOSITORY RECORD}

Zhu, Yongxu, Gan Zheng, Kai-Kit Wong, and Tasos Dagiuklas. 2020. "Spectrum and Energy Efficiency in Dynamic Uav-powered Millimeter Wave Networks”. Loughborough University. https://hdl.handle.net/2134/13608914.v1. 


\title{
Spectrum and Energy Efficiency in Dynamic UAV-Powered Millimeter Wave Networks
}

\author{
Yongxu Zhu, Member, IEEE, Gan Zheng, Senior Member, IEEE, Kai-Kit Wong, Fellow, IEEE, Tasos \\ Dagiuklas, Member, IEEE
}

\begin{abstract}
This letter investigates a dynamic scheme to analyze and optimize unmanned-aerial-vehicle-(UAV)-to-ground millimeter-wave (MMW) networks. First, a downlink energy transfer is proposed, followed by the uplink information transfer process between the UAV base stations (BSs) and a ground Internet of things (IoT) network. The UAVs fly above the ground IoT network in the energy transfer phase with the dynamic regulation to minimize the path loss fading. Afterwards, the ground-based IoT devices use the received energy to transmit the uplink information using vertical links. The UAV BSs are modeled with gain from multiple three-dimensional uniform linear antenna arrays. After deriving new statistical properties, we analyze the spectrum efficiency and energy efficiency for the system. Our numerical results have shown that the dynamic scheme in UAV-to-ground IoT networks provides great advantages than a traditional static deployment.
\end{abstract}

Index Terms-Unmanned aerial vehicles, millimeter wave, wireless power transfer, energy efficiency.

\section{INTRODUCTION}

In the next-generation Internet of Things (IoT) scenarios, the use of low-altitude platforms (LAPs) in aerial wireless communications has become increasingly popular, which has attracted huge attention from both academia and industry. Unmanned Aerial Vehicles (UAVs) benefit from mobility and could act as servers for various IoT devices and networks, for example, data collection, temporary base stations, or energy power beacons [1].

Enhancing the energy performance of UAV systems is becoming the most critical challenge in UAV networks, because UAV wireless networks are limited by the aircrafts' flying speed and weight constraints. The author in [2] has shown that energy-efficient networking schemes in UAV will bring significant benefit when multiple small.Energy harvesting in millimeter-wave (MMW)-based dense networks has been considered in [3]. The energy efficiency in UAV-enabled wireless

Manuscript received Jan 29, 2020, revised May 5, 2020, accepted June 1, 2020. This work was partially supported by the U.K. Engineering and Physical Sciences Research Council (EPSRC) under Grant EP/N007840/1 and EP/N008219/1. The editor coordinating the review of this manuscript and approving it for publication was Dr. Gayan Aruma Baduge. (Corresponding author: Yongxu Zhu)

Y. Zhu and T. Dagiuklas are with the Division of Computer Science and Informatics, London South Bank University, London, SE1 0AA, UK (Email: \{yongxu.zhu, tdagiuklas\}@1sbu.ac.uk).

G. Zheng is with the Wolfson School of Mechanical, Electrical and Manufacturing Engineering, Loughborough University, Leicestershire, LE11 3TU, UK (Email: g.zheng@ @lboro.ac.uk).

K.-K. Wong is with the Department of Electronic and Electrical Engineering, University College London, London, WC1E 6BT, UK (Email: kaikit.wong@ucl.ac.uk). networks has been studied in $[4,5]$. [6] have proposed a UAV-assisted mobile network and analyzed an efficient tradeoff between data collection and energy harvesting to ground devices. The authors in [7] have proposed the harvest-thentransmit protocol in UAV-enabled mobile networks.

The existing literature in $[8,9]$ have focused on moving UAV acting as a wireless charging station in order to serve a limited number of ground IoT devices. Minimization of the total energy consumption in UAV mobile edge computing network with energy harvesting has been investigated in [10]. However, little is known about the wireless communication between multiple UAVs and ground IoT devices in cellular networks, based on the harvest-then-transmit protocol.

In order to tackle this new challenge, stochastic geometry approach has been considered to optimize the effect of multiple MMW antennas on the Spectrum Efficiency (SE) and Energy Efficiency (EE) of UAV-BSs and ground IoT networks, where the UAV-BSs first transfer energy and then collect data from the ground IoT devices. We can apply this network architecture to particular scenarios such as those pertaining to disasters and precision agriculture. To the best of our knowledge, this has not been investigated in the existing literature.

Our main contribution is to investigate a novel dynamic adaptive flight scheme for the wireless power transfer phase along with its detailed mathematical derivation. The proposed scheme can enable UAVs to move above the ground IoT devices and transfer the information, which significantly improves the efficiency of the harvested energy and overcomes the blockage effects to reduce the path loss fading. Moreover, this approach can help the desired UAVs by hovering at an ideal location to achieve better performance at MMW bands due to large path loss. We have also designed a realistic ThreeDimensional (3D) Uniform Linear Array (ULA) beamforming approach to model the UAV-to-ground MMW links after considering the moving elevation and depression angles for typical and interfering UAVs, respectively. From the simulation, we conclude that the proposed scheme is superior to the existing hovering scheme, and the optimal time allocation will maximize both SE and EE.

\section{Network MOdel}

We consider a time-division UAV-to-ground network, which includes multiple UAV BSs having $\mathrm{M}_{o}$ antennas in the air and multiple single-antenna IoT devices deployed on the ground. The locations of all the UAV BSs and ground IoT devices are 


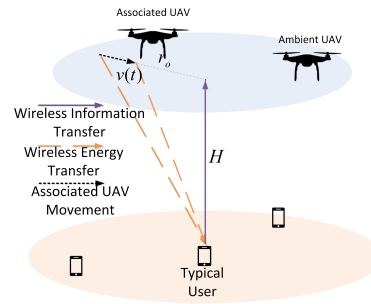

Fig. 1. System model.

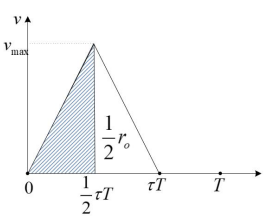

Fig. 2. Velocities and accelerations schematic plot. modeled as a Poisson point process (PPP) configuration in $\Phi_{\mathrm{U}}$ and $\Phi_{\mathrm{S}}$ with densities $\lambda_{\mathrm{U}}$ and $\lambda_{\mathrm{S}}$, respectively, where $\lambda_{\mathrm{S}}$ is far larger than $\lambda_{\mathrm{U}}$. We make the assumption that all the UAV-BSs fly at the same height $H$.

We define $T$ as the duration of a total communication slot, and $\tau \in(0,1)$ as the allocation factor of time. In the first duration, $\tau T$ is allocated to the UAV-to-ground wireless power transfer phase, which includes the time taken for the serving UAV to move to the desired location for that ground IoT device. Typical ground IoT devices first associate with the nearest UAV-BS. Afterwards, the UAV-BS flies towards the typical ground IoT device, with a constant heading rate and altitude, as shown in Fig. 1. During the dynamic adaptive flight process, energy is transmitted to the typical ground IoT device in the downlink phase. The next duration of $(1-\tau) T$ is allocated to the ground-to-UAV wireless information communication phase ${ }^{1}$. A ground IoT device uses the received energy as the transmit power to transmit information back to the associated UAV BSs ${ }^{2}$.

\section{A. Uniform Linear Array}

All the antenna elements are placed along the propagation plane with $\Delta \kappa$ spacing, and we formulate the 3D array steering and response vector $[11,12]$ for the UAV BS as

$\mathbf{u}_{a}^{\mathrm{D}}\left(\theta^{\mathrm{D}}, \vartheta^{\mathrm{D}}, \mathrm{M}_{o}\right)=\left[1, e^{-j \zeta \sin \theta^{\mathrm{D}} \sin \vartheta^{\mathrm{D}}}, . ., e^{-j \zeta\left(\mathrm{M}_{o}-1\right) \sin \theta^{\mathrm{D}} \sin \vartheta^{\mathrm{D}}}\right]^{T}$

where $\theta^{\mathrm{D}} \sim U(0,2 \pi)$ is the azimuth Angle of Departure (AoD) and $\vartheta^{\mathrm{D}} \sim U(0, \pi)$ is the angle of depression for AoD, respectively, and $\zeta=2 \pi \frac{\Delta \kappa}{\varrho}, \varrho$ is the wavelength. $\mathrm{M}_{o}$ is the number of the elements, and $(\cdot)^{T}$ denotes transpose. We obtain the uplink receiving vector $\mathbf{u}_{a}^{\mathrm{A}}\left(\theta^{\mathrm{A}}, \vartheta^{\mathrm{A}}, \mathrm{M}_{o}\right)$ by interchanging $\theta^{\mathrm{D}} \rightarrow \theta^{\mathrm{A}}$ and $\vartheta^{\mathrm{D}} \rightarrow \vartheta^{\mathrm{A}}$ in $(1)$, where $\theta^{\mathrm{A}} \sim U(0,2 \pi)$ is the azimuth Angle of Arrival (AoA) and $\vartheta^{\mathrm{A}} \sim U(0, \pi)$ is the angle of depression for AoA, respectively.

In the ground network, the ground IoTs are only equipped with single-omnidirectional-antenna, which are labeled as $u_{g}=[1]$. Then we have the downlink and uplink ULA steering channel columns given as

$$
\begin{gathered}
\mathbf{U}_{\mathrm{D}}=\mathbf{u}_{a}^{\mathrm{D}}\left(\theta_{o}^{\mathrm{D}}, \vartheta_{o}^{\mathrm{D}}, \mathrm{M}_{o}\right) u_{g}^{H}, \\
\mathbf{U}_{\mathrm{U}}=u_{g}\left[\mathbf{u}_{a}^{\mathrm{A}}\left(\theta_{o}^{\mathrm{A}}, \vartheta_{o}^{\mathrm{A}}, \mathrm{M}_{o}\right)\right]^{H} .
\end{gathered}
$$

Notice $(\cdot)^{H}$ is the conjugate transpose ${ }^{3}$.

\footnotetext{
${ }^{1}$ We consider UAVs with sufficient onboard energy that can be stored for supporting stable transmit power.

${ }^{2}$ Once collected the data from ground IoT devices, each UAV will hover in the current location and wait for another call until the storage space is full.

${ }^{3} \theta_{o}^{\mathrm{A}}$ and $\vartheta_{o}^{\mathrm{A}}$ are the channel angles.
}

It is obvious that the transmitting and receiving array antenna gain from the UAV BS to its serving ground IoT device is $\mathrm{M}_{o}$. Based on Euler's formula, Chebyshev polynomials and trigonometric identities, the average interference antenna gain for downlink and uplink are expressed as

$$
\begin{gathered}
\bar{G}_{\mathrm{D}}=\left|\frac{\mathbf{u}_{a}^{\mathrm{D}}\left(\theta^{\mathrm{D}}, \vartheta^{\mathrm{D}}, \mathrm{M}_{o}\right)^{H}}{\sqrt{\mathrm{M}}_{o}} \mathbf{U}_{\mathrm{D}} \frac{u_{g}^{H}}{1}\right|^{2}=\frac{1}{\mathrm{M}_{o}}\left(\frac{1}{2 \pi}\right)^{2}\left(\frac{1}{\pi}\right)^{2} \\
\times \int_{0}^{\pi} \int_{0}^{2 \pi} \int_{0}^{\pi} \int_{0}^{2 \pi} \frac{1-\cos \left(\mathrm{M}_{o} \zeta \psi_{\mathrm{D}}\right)}{1-\cos \left(\zeta \psi_{\mathrm{D}}\right)} d \theta^{\mathrm{D}} d \vartheta^{\mathrm{D}} d \theta_{o}^{\mathrm{D}} d \vartheta_{o}^{\mathrm{D}} \\
\text { and } \bar{G}_{\mathrm{U}}=\left|\frac{u_{g}^{H}}{1} \mathbf{U}_{\mathrm{U}} \frac{\mathbf{u}_{a}^{\mathrm{A}}\left(\theta^{\mathrm{A}}, \vartheta^{\mathrm{A}}, \mathrm{M}_{o}\right)}{\sqrt{\mathrm{M}_{o}}}\right|^{2}=\frac{1}{\mathrm{M}_{o}} \frac{1}{2 \pi} \frac{1}{\pi} \\
\times \int_{0}^{\pi} \int_{0}^{2 \pi} \frac{1-\cos \left(\mathrm{M}_{o} \zeta \psi_{\mathrm{A}}\right)}{1-\cos \left(\zeta \psi_{\mathrm{A}}\right)} d \theta_{o}^{\mathrm{A}} d \vartheta_{o}^{\mathrm{A}}
\end{gathered}
$$

respectively, where we have defined $\psi_{\mathrm{D}}=\sin \theta^{\mathrm{D}} \sin \vartheta^{\mathrm{D}}-$ $\sin \theta_{o}^{\mathrm{D}} \sin \vartheta_{o}^{\mathrm{D}}$ and $\psi_{\mathrm{A}}=\sin \theta^{\mathrm{A}} \sin \vartheta^{\mathrm{A}}-\sin \theta_{o}^{\mathrm{A}} \sin \vartheta_{o}^{\mathrm{A}}$.

\section{B. Downlink Power Transfer Model}

During the downlink power transmission phase, the associated UAV BS first transmits energy for the duration $\tau T$ with the instantaneous velocity $v(t)(\mathrm{m} / \mathrm{s})$ and constant acceleration $a\left(\mathrm{~m} / \mathrm{s}^{2}\right)$ toward the typical ground IoT device. Considering blockage effects in the wireless channel of the UAV-to-ground network, different path loss probabilities have been applied to Line-of-Sight (LoS) links and Non-Line-of-Sight (NLoS) links. Due to the sparse scattering in the MMW channel, we have neglected small scale fading [13].

The total harvested energy from associated UAV BS and ambient UAV BSs to the ground IoT device as

$$
\mathcal{E}_{\text {sum }}=\eta \underbrace{\frac{\tau T P_{\mathrm{U}} \mathrm{M}_{o} \beta}{\mathcal{D}\left(\left|X_{o}(t)\right|\right)}}_{\mathcal{E}_{1}}+\eta \underbrace{\tau T \sum_{q \in \Phi_{\mathrm{U}}} \frac{P_{\mathrm{U}} \beta \bar{G}_{\mathrm{D}}}{\mathcal{D}\left(\left|X_{q}(t)\right|\right)}}_{\mathcal{E}_{2}},
$$

, where $\mathcal{E}_{1}$ is the directed energy from the associated UAV, $P_{\mathrm{U}}$ is the transmitting power from all the UAV BSs and $X_{o}(t)$ is the instant projection distance between the moving associated UAV BS to the typical IoT device. Notably, the horizontal distance $X_{o}(t)=r_{o}-s(t)$ is dynamic, i.e., varying with time $t$, where $t \in[0, \tau T], r_{o}$ is the initial closest associationprojection distance from UAV to typical IoT devices based on the associated probability density functions, which is given by $f\left(r_{o}\right)=2 \pi \lambda_{\mathrm{U}} r_{o} \exp \left(-\pi \lambda_{\mathrm{U}} r_{o}^{2}\right)$ [14]. $\mathcal{E}_{2}$ is the harvested energy from the ambient UAVs, and $X_{q}(t)$ is the dynamic projection distances between typical ground IoT device and the ambient wireless power charging UAV BSs which varies with time $t . \eta$ is the energy conversion efficiency factor, and $\beta$ is the frequency-dependent constant value.

The path loss gain function can be expressed by

$$
\mathcal{D}(X)=\frac{p_{\mathrm{L}}(X)}{{\sqrt{X^{2}+H^{2}}}^{\alpha_{\mathrm{L}}}}+\frac{p_{\mathrm{N}}(X)}{{\sqrt{X^{2}+H^{2}}}^{\alpha_{\mathrm{N}}}},
$$

where $X$ indicates the projection distance from the serving UAV to the typical user, $\alpha_{\mathrm{L}}$ and $\alpha_{\mathrm{N}}$ are the LoS and NLoS path loss exponents, respectively. $p_{\mathrm{L}}(X)$ is the LoS connection probability, while the NLoS probability of a link is $p_{\mathrm{N}}(X)=$ $1-p_{\mathrm{L}}(X)$. 


\section{Uplink Information Transmission}

The received Signal-to-Interference-plus-Noise Ratio (SINR) of the serving UAV BS with distance $\left|X_{o}\right|$ is given by

$$
\gamma=\frac{P_{\mathrm{T}} \mathrm{M}_{o} \beta \mathcal{D}\left(\left|\widehat{X}_{o}\right|\right)}{\sum_{k \in \Phi_{\mathrm{U}} \backslash o} P_{\mathrm{T}} \bar{G}_{\mathrm{U}} \beta \mathcal{D}\left(\left|Z_{k}\right|\right)+\sigma^{2}},
$$

where $P_{\mathrm{T}}$ is the maximum stable transmit power from the ground IoT device, and $\widehat{X}_{o}$ is the projection distance between the serving UAV BS and its intended IoT device, $\bar{G}_{\mathrm{U}}$ is the uplink array gain and $Z_{k}$ is the projection distance between the typical ground IoT device and $k$-th interfering UAV BSs, and $\sigma^{2}$ is the noise power.

\section{Performance AnAlysis}

In this section, the downlink average harvested energy and average achievable rate in the uplink are analysed.

\section{A. UAV-to-ground Wireless Power Transfer}

In the wireless power transfer phase, each typical ground IoT device is associated with the nearest UAV BS. The associated UAV BS flies towards the serving ground IoT device whilst transmitting energy.

Note that we assumed that each associated UAV flies at constant acceleration as in the beginning. Then, each UAV is stopped right above the typical user and remained aloft, so that the minimum path-loss fading between the typical IoT device and serving UAV in the information transmission process is guaranteed. In the first phase (i.e., from a hovering state to maximum velocity), an adaptive and dynamical solution has been designed where UAVs fly at constant acceleration to achieve the maximum velocity. Then, the UAVs decelerate to the destination, stopping and hovering on the top of the typical IoT device during the second duration (i.e., from the maximum velocity to hovering state). For further analysis, we calculated the instant velocity and constant acceleration has been calculated, which was based on the given distance $r_{o}$ expression as

$$
\left\{\begin{array}{ll}
v_{1}\left(r_{o}, \tau, t\right)=4 r_{o} /(\tau T)^{2} t<v_{\mathrm{m}} & 0 \leqslant t \leqslant \frac{\tau T}{2} \\
v_{2}\left(r_{o}, \tau, t\right)=\left(4 r_{o}-4 r_{o} / \tau T\right) /(\tau T)<v_{\mathrm{m}} & \frac{\tau T}{2} \leqslant t \leqslant \tau T
\end{array},\right.
$$

where the acceleration can be expressed as

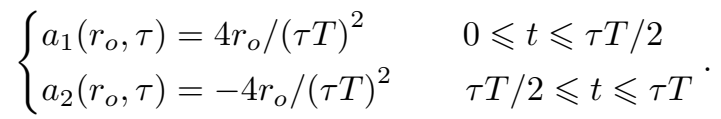

Note that $v_{\mathrm{m}}=a_{\mathrm{m}} \tau T / 2$ is the constant for the velocity. $0<$ $a_{1}\left(r_{o}, \tau\right)<a_{\mathrm{m}}$ and $-a_{\mathrm{m}}<a_{2}\left(r_{o}, \tau\right)<0$ are the acceleration and the deceleration along the UAV's flying direction, $a_{\mathrm{m}}$ and $-a_{\mathrm{m}}$ are the constant for the acceleration and deceleration, respectively.

Theorem 1: The average harvested energy for the associated typical IoT device served by the UAV-BSs with the power transfer time allocation $\tau$ is given by (11) on the top of this page. Note that the azimuth $\mathrm{AoD} \theta^{\mathrm{D}}$ and depression $\mathrm{AoD} \vartheta^{\mathrm{D}}$ from the associated UAV BS will change as the UAV flies, which adapt to the moving direction as the UAV BS flies.

Proof 1: Based on (11), the average harvested energy for a typical IoT device projection distance is written as

$$
\mathcal{E}_{\text {sum }}\left(r_{o}, \tau\right)=\eta\left\{\mathcal{E}_{1}\left(r_{o}, \tau\right)+\mathcal{E}_{2}\left(r_{o}, \tau\right)\right\} .
$$

The average harvested energy directly from the associated UAV BS can be written as

$$
\begin{array}{r}
\mathcal{E}_{1}\left(r_{o}, \tau\right)=P_{\mathrm{U}} \mathrm{M}_{o} \beta\left\{\int_{0}^{\tau T / 2} \mathcal{D}\left(r_{o}-s_{1}\left(r_{o}, \tau, t\right)\right) d t\right. \\
\left.+\int_{\tau T / 2}^{\tau T} \mathcal{D}\left(r_{o}-s_{2}\left(r_{o}, \tau, t\right)\right) d t\right\} \\
\text { with } \quad s_{1}\left(r_{o}, \tau, t\right)=\frac{2 r_{o}}{(\tau T)^{2}} t^{2}, \\
s_{2}\left(r_{o}, \tau, t\right)=\frac{4 r_{o}}{(\tau T)} t-\frac{2 r_{o}}{(\tau T)^{2}} t^{2}-r_{o},
\end{array}
$$

where $s_{1}$ and $s_{2}$ are referred to the moving distance in the straight line during first phase and second phase, respectively, and $r_{o}$ is the distance between the staring point to the destination. To avoid the low efficiency association link and high moving cost, we prevent the association distance $r_{o}$ larger than $r_{\mathrm{d}}(\tau)=a_{\mathrm{m}}(\tau T)^{2} / 4$, where the associated UAV can not fly to the top of the typical user.

The ambient harvested energy $\mathcal{E}_{2}$ under the prescribed association distance and time allocation is calculated as

$$
\begin{aligned}
& \mathcal{E}_{2}(\tau, r)=\tau T 2 \pi \lambda_{\mathrm{U}} P_{\mathrm{U}} \bar{G}_{D} \beta \int_{0}^{\infty} \int_{0}^{2 \pi} \times \\
& \left\{\int_{0}^{\frac{\tau T}{2}} \mathcal{D}\left(U_{1}(\phi, t, u)\right) u d t+\int_{\frac{\tau T}{2}}^{\tau T} \mathcal{D}\left(U_{2}(\phi, t, u)\right) u d t\right\} d \phi d u \\
& \stackrel{(a)}{\approx} \tau T 2 \pi \lambda_{\mathrm{U}} \int_{0}^{\infty} P_{\mathrm{U}} \bar{G}_{D} \beta \mathcal{D}(u) u d u
\end{aligned}
$$

where $U_{1}$ and $U_{2}$ are the instant projection distance from interference UAV to the typical IoT device in the first and second phase at time $t$, respectively.

$$
\begin{aligned}
& U_{1}(\phi, t, u)=\sqrt{u^{2}+s_{1}\left(\bar{u}_{l}, \tau, t\right)^{2}-2 u s_{1}\left(\bar{u}_{l}, \tau, t\right) \cos \phi}, \\
& U_{2}(\phi, t, u)=\sqrt{u^{2}+s_{2}\left(\bar{u}_{l}, \tau, t\right)^{2}-2 u s_{2}\left(\bar{u}_{l}, \tau, t\right) \cos \phi}
\end{aligned}
$$

where $s_{1}\left(\bar{u}_{l}, \tau, t\right)$ and $s_{2}\left(\bar{u}_{l}, \tau, t\right)$ are obtained by interchanging the parameters $r_{o} \rightarrow \bar{u}_{l}$ in (14), and $u$ is the projection distance from interference UAV to the typical IoT device. $\phi$ is the inclined angle with original interference UAV to the typical IoT device and the interference UAV to the destination IoT device. We simply use the average nearest distance to replace the dynamic distance from the interference UAV to its serving IoT device as $\bar{u}_{l}=\int_{0}^{\infty} r_{o} f\left(r_{o}\right) d r_{o}$.

Note that the approximation $(a)$ in (15) is obtained by assuming each interference UAV flies to the destination UAV with random inclined angle $\phi$, which will counteract the impact of mobility. Moreover, consider the interference only has negligible effect on MMW links, then we can obtain it 


$$
\begin{aligned}
\mathcal{E}_{\text {sum }}\left(r_{o}, \tau\right) & =\eta \tau T 2 \pi \lambda_{\mathrm{U}} P_{\mathrm{U}} \bar{G}_{\mathrm{D}} \beta \int_{0}^{\infty} \mathcal{D}(u) u d u \\
& +\eta P_{\mathrm{U}} \mathrm{M}_{o} \beta\left\{\int_{0}^{\frac{\tau T}{2}} \mathcal{D}\left(r_{o}\left(1-2\left(\frac{t}{\tau T}\right)^{2}\right)\right) d t+\int_{\frac{\tau T}{2}}^{\tau T} \mathcal{D}\left(r_{o}\left(2-4 \frac{t}{\tau T}+2\left(\frac{t}{\tau T}\right)^{2}\right)\right) d t\right\} .
\end{aligned}
$$

by approximating the generating function of PPP [15]. By applying (13) and (15) into (12), we obtain the desired result in (11).

\section{B. Ground-to-UAV Wireless Information Communication}

After the energy transfer phase, the IoT devices transmit their information signals to the associated UAV BSs ${ }^{4}$. Since the uplink information transmission energy consumed could not exceed the received energy $\mathcal{E}_{\text {sum }}$, and the average transmit power in the ground IoT device is $P_{\mathrm{T}}\left(r_{o}, \tau\right)=$ $\mathcal{E}_{\text {sum }}\left(r_{o}, \tau\right) /(1-\tau) T$, where $\mathcal{E}_{\text {sum }}\left(r_{o}, \tau\right)$ is given by (11).

Theorem 2: For a UAV BS associated with its intended ground IoT device, the average achievable rate at each UAV BS can be found as

$$
\begin{aligned}
\mathcal{R}\left(r_{o}, \tau\right) & =\frac{(1-\tau) T}{\ln 2} \int_{0}^{\infty} \frac{1}{w}\left(1-\exp \left(-w \mathrm{M}_{o} \beta H^{-\alpha_{\mathrm{L}}}\right)\right) \\
& \times \exp \left(-w 2 \pi \lambda_{\mathrm{U}} \bar{G}_{\mathrm{U}} \beta \int_{0}^{\infty} \mathcal{D}(v) v d v\right) \\
& \times \exp \left(-w \frac{\sigma^{2}}{P_{\mathrm{T}}\left(r_{o}, \tau\right)}\right) d w
\end{aligned}
$$

Proof 2: The average uplink rate from the typical ground IoT device to the associated UAV BS can be expressed as

$$
\begin{aligned}
\mathcal{R}\left(r_{o}, \tau\right) & =\frac{(1-\tau) T}{\ln 2} \int_{0}^{\infty} \frac{1}{w}\left(1-e^{-w \gamma}\right) d w=\frac{(1-\tau) T}{\ln 2} \\
& \times \int_{0}^{\infty} \frac{1}{w}(1-\underbrace{e^{-w S}}_{\Theta_{S}(w)}) \underbrace{e^{-w I}}_{\Theta_{I}(w)} e^{-\frac{w \sigma^{2} \tau T}{\overline{\mathcal{S}}_{\operatorname{sum}}\left(r_{o}, \tau\right)}} d w .
\end{aligned}
$$

Since the received signal and interference are independent, we derive the signal expression as $\Theta_{S}(w)=$ $\exp \left(-w \mathbf{M}_{o} \beta H^{-\alpha_{\mathrm{L}}}\right)$, and then $\Theta_{I}(w)$ is calculated as

$$
\begin{aligned}
\Theta_{I}(w) & =\mathbb{E}\left[\exp \left(-w \sum_{k \in \Phi_{\mathrm{U}}} \bar{G}_{U} \beta{\sqrt{u_{k}^{2}+H^{2}}}^{-\alpha_{\mathrm{U}}}\right)\right] \\
& \approx \exp \left\{-w 2 \pi \lambda_{\mathrm{U}} \bar{G}_{\mathrm{U}} \beta \int_{0}^{\infty} \mathcal{D}(v) v d v\right\} .
\end{aligned}
$$

Substituting $\Theta_{S}(w)$ and $\Theta_{I}(w)$ into (18), we obtain the desired result (17).

\section{Energy Efficiency}

The energy efficiency of the overall UAV-to-ground downlink and uplink system is defined as the ratio of the average achievable rate to the total average power consumption. We consider the total average power consumption composed of the energy transfer phase and the information transfer phase,

\footnotetext{
${ }^{4}$ It is assumed that the association pair is the same as in the downlink phase.
}

which includes flying power and hovering power consumption, respectively. This problem can be formulated as

$$
\mathbb{P}: \max _{\tau} \mathcal{B}_{\mathrm{EE}}^{\mathrm{Low}}\left(\tau, r_{o}\right)=\int_{0}^{r_{\mathrm{m}}(\tau)} \frac{R\left(r_{o}, \tau\right)}{\mathcal{E}_{\mathrm{F}}\left(r_{o}, \tau\right)+\mathcal{E}_{\mathrm{O}}(\tau)} f\left(r_{o}\right) d r_{o},
$$

where the average flying energy $\mathcal{E}_{\mathrm{F}}\left(r_{o}, \tau\right)$ is given as

$$
\begin{gathered}
\mathcal{E}_{\mathrm{F}}\left(r_{o}, \tau\right)=\int_{0}^{\frac{\tau T}{2}} k_{1} v_{1}\left(r_{o}, \tau, t\right)^{3}+\frac{k_{2}\left(1+\left|a\left(r_{o}\right)\right|^{2} / g^{2}\right)}{v_{1}(t)} d t \\
\quad+\int_{\frac{\tau T}{2}}^{\tau T} k_{1} v_{2}\left(r_{o}, t\right)^{3}+\frac{k_{2}\left(1+\left|a\left(r_{o}\right)\right|^{2} / g^{2}\right)}{v_{2}\left(r_{o}, t\right)} d t
\end{gathered}
$$

where $k_{1}$ and $k_{2}$ are the constant values and $g$ is the gravitation acceleration with the nominal value is $9.8 \mathrm{~m} / \mathrm{s}^{2}$. The remaining consumption energy is $\mathcal{E}_{\mathrm{O}}(\tau)=\tau T P_{\mathrm{U}}+(1-\tau) T P_{\mathrm{H}}$, while $P_{\mathrm{H}}$ is the hovering power which ensures that the UAV remains aloft [5]. We formulate the optimization problem to find the optimal time allocation of $\tau$ to maximize the average energy efficiency. Since the optimization of the energy efficiency $\mathcal{B}_{\mathrm{EE}}$ for time allocation $\tau$ does not permit a closed-form solution, we solve the problem by using a simple one-dimensional search in the numerical results.

\section{Numerical Results and Discussions}

In this section, we present numerical results of UAV to ground channel characteristics and multiple 3D ULA antenna array on the achievable energy efficiency. We assume the bandwidth with $\mathrm{BW}=1 \mathrm{GHz}$, where the pathloss exponent are $\alpha_{\mathrm{L}}=2.5$ and $\alpha_{\mathrm{N}}=3.71$, respectively. The noise figure is $\mathrm{N}_{f}=10 \mathrm{~dB}$, the noise power is $\sigma^{2}=-174+$ $10 \log 10(\mathrm{BW})+\mathrm{N}_{f} \mathrm{dBm}$. Then we have the frequency dependent constant value given by $\beta=\left(\frac{c}{4 \pi f_{c}}\right)^{2}$ with $c=$ $3 \times 10^{8} \mathrm{~m} / \mathrm{s}$. We also assume that $k_{1}=9.26 \times 10^{-4}$ and $k_{2}=2.25 \times 10^{3}$ in the constant for the UAV flying power [5], $a_{\mathrm{m}}=4 \mathrm{~m} / \mathrm{s}^{2}, \eta=0.9, P_{\mathrm{U}}=46 \mathrm{dBm}, \mathrm{M}_{o}=16, P_{\mathrm{H}}=80 \mathrm{~W}$. In the ULA configuration, we choose the antenna spacing as half wavelength $\Delta \kappa=\frac{1}{2} \varrho$.

The blockages are modeled as [16], and the LoS connection probability function in a network is computed as

$$
p_{\mathrm{L}}(X)=1+a \exp (-b[\arctan (H / X)-a]),
$$

where $a=9.6$ and $b=0.28$, respectively. For comparison, we consider the static hovering scheme, in which all the UAVs hover at their original location and do not fly to the IoT devices.

Fig. 3 shows the energy efficiency against the time allocation of $\tau$. We observe that as the time allocation $\tau$ increases, there exists an optimal value of $\tau$ which achieves 

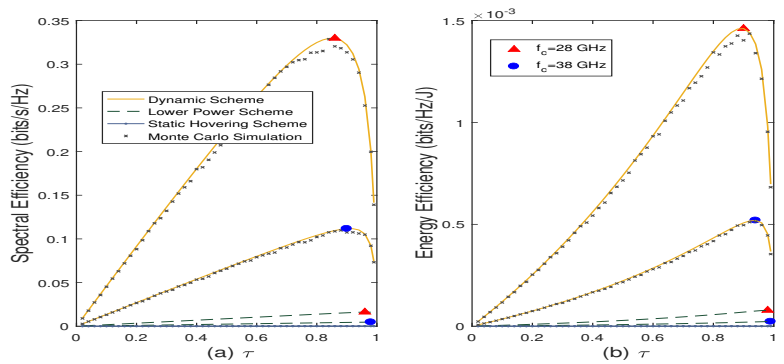

Fig. 3. SE and $\mathrm{EE}$ vs the time allocation, where $H=5 \mathrm{~m}$, $\lambda_{\mathrm{U}}=100 / \mathrm{km}^{2}, T=5 \mathrm{~s}$.
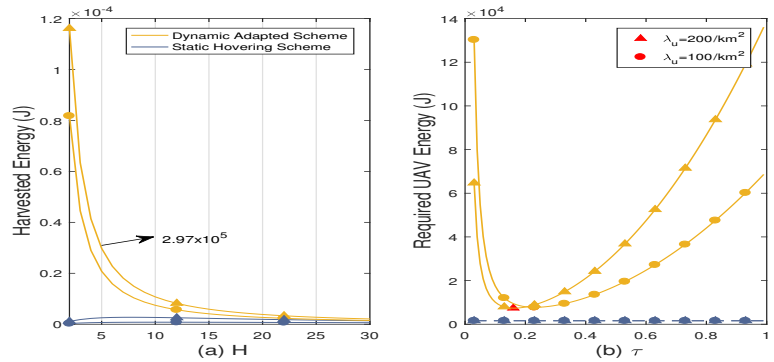

Fig. 4. Harvested energy vs height, and required UAV energy vs time allocation, where $T=20 \mathrm{~s}, \tau=0.7$ in (a), $f_{c}=38$ $\mathrm{GHz}$.

the maximum $\mathrm{SE}$ and $\mathrm{EE}$, and this optimal value changes with frequency when $f_{c}=28 \mathrm{GHz}$ and $f_{c}=38 \mathrm{GHz}$, respectively. Furthermore, our dynamic scheme shows superiority to the static hovering scheme, due to a dynamic adaptive scheme effectively eliminate blockage effects in the information transfer phase. For further comparison, we examined a new Lower power scheme, which uses the transmit power from the static hovering scheme to replace the dynamic scheme. The results show that the dynamic adaptive scheme not only benefits the wireless power transfer phase, it also brings benefits in the information transfer phase. As expected, the simulation results for EE provide similar insights to those for SE.

Fig. 4 (a) presents the harvested energy curve versus height $H$. We can observe that as the height increases, the harvested energy demonstrates a significant reduction for the proposed scheme. In contrast, the hovering scheme can harvest extremely lower energy than the dynamic scheme. Fig. 4 (b) presents the required UAV energy curve versus time allocation $\tau$. The required energy includes mechanical energy and transmit energy. We observe that the dynamic scheme consumes more energy than the static hovering scheme and the optimal time allocation $\tau$ that minimized the energy required are 0.16 and 0.22 when $\lambda_{u}=200 / \mathrm{km}^{2}$ and $\lambda_{u}=100 / \mathrm{km}^{2}$, respectively.

Overall, the dynamic scheme consumes more propulsion energy than the hovering scheme. However, the dynamic adaptive scheme also harvests more energy and demonstrate superiority over traditional static networks in both SE and EE. The proposed scheme is significantly advantageous for both the wireless power transfer and information transfer phases.

\section{CONClusions And Future Work}

This letter has proposed an improved dynamic process for downlink wireless power transfer and uplink information transfer architecture for UAV-to-ground networks. Based on the adaptive flying scheme, each link minimizes path loss fading and avoids blockage effects. We have considered SE and EE in order to evaluate the performance of this network. The numerical results indicated that although the proposed moving scheme consumed considerable energy, it still demonstrated considerable advantages for $\mathrm{SE}$ and $\mathrm{EE}$ as compared to the conventional static hovering network. Besides, optimizing the time allocation can maximize SE and EE, respectively. We expect future advances to reduce the energy consumption of UAV flights and to design a tradeoff between EE and SE.

\section{REFERENCES}

[1] N. H. Motlagh, T. Taleb, and O. Arouk, "Low-altitude unmanned aerial vehicles-based internet of things services: Comprehensive survey and future perspectives," IEEE Internet of Things J., vol. 3, no. 6, pp. 899922, Dec. 2016.

[2] J. Wang, C. Jiang, Z. Han, Y. Ren, R. G. Maunder, and L. Hanzo, "Taking drones to the next level: Cooperative distributed UnmannedAerial-Vehicular networks for small and mini drones," IEEE Veh. Technol. Mag., vol. 12, no. 3, pp. 73-82, Sep. 2017.

[3] H. Zhang, S. Huang, C. Jiang, K. Long, V. C. M. Leung, and H. V. Poor, "Energy efficient user association and power allocation in millimeterwave-based ultra dense networks with energy harvesting base stations," IEEE J. Sel. Areas Commun., vol. 35, no. 9, pp. 1936-1947, Sep. 2017.

[4] Y. Zeng, J. Xu, and R. Zhang, "Energy minimization for wireless communication with rotary-wing UAV," IEEE Trans. Wireless Commun., vol. 18, no. 4, pp. 2329-2345, April 2019.

[5] Y. Zeng and R. Zhang, "Energy-efficient UAV communication with trajectory optimization," IEEE Trans. Wireless Commun., vol. 16, no. 6 , pp. 3747-3760, June 2017.

[6] L. Ruan, J. Chen, Q. Guo, H. Jiang, Y. Zhang, and D. Liu, "A coalition formation game approach for efficient cooperative multi-UAV deployment," Applied Sciences, vol. 8, no. 12, p. 2427, May 2018.

[7] Z. Yang, W. Xu, and M. Shikh-Bahaei, "Energy efficient uav communication with energy harvesting," IEEE Trans. Veh. Technol., vol. 69, no. 2, pp. 1913-1927, 2020.

[8] J. Xu, Y. Zeng, and R. Zhang, "UAV-enabled wireless power transfer: Trajectory design and energy optimization," IEEE Trans. Wireless Commun., vol. 17, no. 8, pp. 5092-5106, Aug 2018.

[9] A. Ali and M. O. Hasna, "Energy harvesting schemes for uav based communications," in 2019 16th IEEE Annual Consumer Communications Networking Conference (CCNC), Jan 2019, pp. 1-2.

[10] Z. Yang, C. Pan, K. Wang, and M. Shikh-Bahaei, "Energy efficient resource allocation in uav-enabled mobile edge computing networks," IEEE Trans. Wireless Commun., vol. 18, no. 9, pp. 4576-4589, 2019.

[11] A. H. Tewfik and W. Hong, "On the application of uniform linear array bearing estimation techniques to uniform circular arrays," IEEE Trans. Signal Process., vol. 40, no. 4, pp. 1008-1011, Apr. 1992.

[12] N. Moraitis and P. Constantinou, "Indoor channel capacity evaluation utilizing ULA and URA antennas in the millimeter wave band," in 2007 IEEE 18th International Symposium on Personal, Indoor and Mobile Radio Communications, Sep. 2007, pp. 1-5.

[13] T. S. Rappaport, S. Sun, R. Mayzus, H. Zhao, Y. Azar, K. Wang, G. N. Wong, J. K. Schulz, M. Samimi, and F. Gutierrez, "Millimeter wave mobile communications for $5 \mathrm{~g}$ cellular: It will work!" IEEE Access, vol. 1, pp. 335-349, 2013.

[14] Y. Zhu, L. Wang, K. Wong, S. Jin, and Z. Zheng, "Wireless power transfer in massive mimo-aided hetnets with user association," IEEE Trans. Commun., vol. 64, no. 10, pp. 4181-4195, 2016.

[15] F. Baccelli, B. Błaszczyszyn et al., "Stochastic geometry and wireless networks: Volume II Applications," Foundations and Trends $®$ in Networking, vol. 4, no. 1-2, pp. 1-312, Jan. 2010.

[16] M. Mozaffari, W. Saad, M. Bennis, and M. Debbah, "Drone small cells in the clouds: Design, deployment and performance analysis," in 2015 IEEE Global Communications Conference (GLOBECOM), Dec 2015, pp. 1-6. 